\title{
«Estudiar no, pero integrarse... estupendamente»: alteridad y alumnado inmigrante denominado "latinoamericano" en la escuela española
}

Antonia Olmos Alcaraz Universidad de Granada (España). Departamento de Antropología Social. Instituto de Migraciones

María Rubio Gómez ONG Prodiversa (Progreso y Diversidad). Instituto de Migraciones. Universidad de Granada (España)

\section{Introducción}

La inmigración forma parte de la realidad española desde hace décadas. Fue a principios de los años noventa cuando empezó a ser notable estadísticamente la llegada de inmigrantes al país. A partir de entonces el fenómeno ha sido representado por medios de comunicación, por discursos políticos y por la sociedad en su conjunto como una problemática social de primer orden (Olmos-Alcaraz, 2008; Granados, 2013; Van Dijk, 2009). Así mismo ha sido gestionado en tanto que problemática, en lo relativo a gestión de flujos y a políticas de integración social (Zapata-Barrero, 2001; Olmos-Alcaraz, 2009; García y Olmos-Alcaraz, 2010). Esta forma de entender las migraciones se ha 
mantenido estable durante los últimos 25 años en España, no obstante los discursos y las prácticas políticas durante la época de crecimiento económico del país fueron de corte más utilitarista/funcionalista (Olmos-Alcaraz, 2009); y en el momento actual se han vuelto más explícitamente diferenciadoras y racistas (Sebastiani, 2015).

El fenómeno se hizo presente en las escuelas poco después de iniciarse su reconocimiento en las estadísticas oficiales, a mediados de la década de los noventa especialmente. Es así como llegan los hijos e hijas de inmigrantes a la escuela española: de distintas procedencias, de distintas nacionalidades, con distintas características culturales. Los escolares "latinoamericanos" (América Sur y Centro) suponen un volumen considerable entre dicha población, si bien no son la más numerosa entre el alumnado de nacionalidades extranjeras en el país (para el curso 2014-15, representan 27,8 \%, por detrás de aquellos que ostentan nacionalidad de algún país africano, según datos del Ministerio de Educación, Cultura y Deporte). Tampoco son destinatarios directos de atención de políticas educativas, dado que en España, estas están centradas fundamentalmente en cuestiones lingüísticas (García et al., 2007; Olmos-Alcaraz, 2010; Rubio-Gómez, 2013). Siendo así, los imaginarios sociales que se construyen sobre los mismos comparten lógicas de funcionamiento con las representaciones que sobre el fenómeno migratorio en general funcionan en el país: el inmigrante como "pobre", el inmigrante como "extraño", distinto y distante culturalmente, el inmigrante como "delincuente", etc. (Olmos-Alcaraz, 2009); pero, por otro lado, adquieren características que le son propias (Rubio-Gómez, 2013): la percepción de "cercanía” y los imaginarios coloniales que siguen presentes y son re-interpretados en el contexto actual globalizado. Estas representaciones, y el cómo las mismas entran en interacción con otras formas de alteridad referida a la población inmigrante, nos dan las claves para entender las lógicas de construcción de la diferencia existentes en España con respecto a dichos movimientos migratorios.

El objetivo de este texto es analizar las lógicas de construcción de alteridad de la población de origen latinoamericano en la escuela española. Para ello, en primer lugar, ofrecemos una descripción de la metodología seguida en nuestra investigación; a continuación incluimos datos 
estadísticos sobre la población de origen latinoamericano en España, en general, y en espacios educativos, en particular; en tercer lugar analizamos las representaciones sociales sobre "lo latino" en la escuela española; finalizamos este trabajo con una reflexión sobre las lógicas de construcción de la diferencia en la escuela y su relación con las nuevas lógicas de funcionamiento del racismo en el contexto estudiado.

\section{Metodología}

La metodología que hemos seguido para llevar a cabo nuestro trabajo es de carácter etnográfico. La etnografía escolar supone la forma más habitual de realizar investigación actualmente en el campo de la Antropología de la Educación (Serra, 2004; Jociles, 2007; García y Pulido, 2008), y es aquí donde se sitúan nuestras investigaciones ${ }^{1}$. Las mismas han sido llevadas a cabo en los últimos años en centros educativos andaluces (España), en los niveles de educación primaria y secundaria, y nos han permitido generar los datos que aquí presentamos. Hemos analizado prácticas y discursos fruto de nuestra observación y la realización de entrevistas en los centros educativos visitados. En la Tabla $\mathrm{N}^{\mathrm{o}} 1$ pueden verse las entrevistas llevadas a cabo y los perfiles de las personas entrevistadas.

1 Las investigaciones son "Construyendo diferencias en la escuela. Estudios de las trayectorias de las ATAL en Andalucía, de su profesorado y de su alumnado” (Ministerio de Ciencia e Innovación, Plan Nacional de I+D+i, 2014-2016), "Éxitos y fracasos escolares. Trayectorias socioculturales de inmigrantes extranjeros y escolares autóctonos en el sistema educativo andaluza" (Ministerio de Ciencia e Innovación, Plan Nacional de I+D+i, 2010-2013), “Multiculturalidad e integración de la población inmigrante extranjera en las escuelas andaluzas" (Junta de Andalucía, Proyectos de Excelencia, 2007-2010) y "Evaluación de las actuaciones de atención educativa del alumnado inmigrante en Andalucía, Junta de Andalucía, 2004-2006). 
Tabla 1. Entrevistas realizadas

\begin{tabular}{|c|c|c|}
\hline \multirow{2}{*}{$\begin{array}{l}\text { Perfil profesional de las } \\
\text { personas entrevistadas }\end{array}$} & \multicolumn{2}{|c|}{$\mathbf{N}^{\mathrm{o}}$ de entrevistas realizadas } \\
\hline & $\begin{array}{c}\text { Educación } \\
\text { primaria }\end{array}$ & $\begin{array}{l}\text { Educación } \\
\text { secundaria }\end{array}$ \\
\hline $\begin{array}{c}\text { Equipo directivo (directores, } \\
\text { subdirectores, jefes de estudios y } \\
\text { secretarios) }\end{array}$ & 49 & 50 \\
\hline Profesorado general & 5 & 15 \\
\hline Orientadores & \multicolumn{2}{|c|}{14} \\
\hline Profesorado de ATAL ${ }^{2}$ & \multicolumn{2}{|c|}{41} \\
\hline Profesorado de $\mathrm{ELCO}^{3}$ & \multicolumn{2}{|c|}{2} \\
\hline
\end{tabular}

Fuente: elaboración propia de las autoras.

La cantidad de centros educativos visitados a lo largo de nuestras investigaciones ha sido importante (44 de educación secundaria y 47 de educación primaria), si bien la intensidad de trabajo en cada uno de ellos ha sido variable. Siendo así, los centros situados en las provincias de Málaga, Almería (las de mayor porcentaje de población extranjera en la región) y Granada (donde se sitúa nuestro lugar de trabajo) han sido más intensamente explorados.

Veamos a continuación de forma más detallada algunas cifras sobre extranjería en España y sobre escolarización de alumnado extranjero en el sistema educativo español, para dar un mayor contexto a nuestros datos de campo.

2 ATAL es la sigla de Aulas Temporales de Adaptación Lingüística. Da nombre a una de las más populares medidas de atención a la diversidad instaurada en las escuelas de Andalucía, centrada en enseñar la lengua vehicular de la escuela al alumnado que la desconoce.

3 Enseñanza de Lengua y Cultura de Origen. Se trata de programas que se llevan a cabo fuera del horario escolar, y cuyo profesorado pertenece a -y es financiado por- la comunidad de origen del alumnado inmigrado de nacionalidad extranjera. 


\section{Evolución de las cifras de extranjería y escolarización de alumnado de nacionalidad extranjera en España}

La forma de medir los flujos migratorios no es sencilla: diferentes fuentes estadísticas, diferentes formas de medir en cada una de ellas y distintos tipos de datos como resultado. Por otro lado no siempre está claro cuándo se está hablando de "estimaciones" (los flujos no pueden ser otra cosa, dado que están en continuo movimiento), de datos tipo stock o cuándo se está hablando de extranjería y no de inmigración (aunque ambas situaciones o procesos puedan coincidir en ocasiones). Siendo así ofrecemos datos sobre extranjería -para hablar de migraciones- no sin asumir los riesgos que ello pueda conllevar. Por otro lado hemos de aclarar que tratamos de dar datos estadísticos sobre categorías socio-culturales (la de "latinoamericano" es una de ellas), con todos los reduccionismos que ello pueda implicar. Un ejemplo de ello lo observamos al descubrir cómo, muy frecuentemente, desde las instituciones oficiales se contabiliza la extranjería agrupando las cifras por continentes. En este sentido para hablar de población extranjera denominada "latinoamericana" en España hemos de hablar de población extranjera con alguna nacionalidad procedente de Sur y Centro América; sin embargo, sabemos que -y especialmente cuando hablamos de jóvenes en edad escolar- muchos de los sujetos que son objeto de categorización con la etiqueta "latino" o "latinoamericano" no van a tener nacionalidad extranjera, van a ser españoles, y algunos incluso no serán inmigrantes al haber nacido ya en España: son las mal llamadas "segundas generaciones" 4

Realizadas estas aclaraciones pasamos a describir los datos comentados. A continuación, en la Tabla 2, podemos ver la evolución de la población extranjera en España desde el año 2000 hasta la actualidad:

4 Dicha categoría social parece sugerir que una acción (migrar) se "hereda", "se transmite" de generación en generación, y con ella todos los significados culturales asociados a la misma. 
Tabla 2. Evolución población extranjera en España (2000-2015)

\begin{tabular}{|c|c|c|}
\hline Año & Número & $\%$ \\
\hline 2000 & 895.720 & 2,2 \\
\hline 2005 & $2 ’ 738.932$ & 6,2 \\
\hline 2010 & $4 ' 926.608$ & 10,5 \\
\hline 2015 & $4 ' 447.852$ & 9,6 \\
\hline
\end{tabular}

Fuente: elaboración propia a partir de datos del Instituto Nacional de Estadística.

Como se puede observar desde principios del presente siglo la tendencia siempre había sido de crecimiento de los efectivos, pero en los últimos cinco años por primera vez se constata cómo los mismos no solo dejan de aumentar sino que decrecen. Hay varias explicaciones al respecto. La más sencilla, no sabemos si también la que más se ajusta a la realidad, es argumentar que han dejado de llegar personas extranjeras al país y además muchas de las que estaban en él han regresado a sus países de origen. Sin embargo esta disminución del porcentaje de población extranjera residente en España se debe también a las nacionalizaciones, que son ahora -por motivos lógicos como presencia más antigua en el territorio- más numerosas que en años anteriores.

$\mathrm{Si}$ atendemos a las procedencias por nacionalidades, según el Observatorio Permanente de la Inmigración, los datos entre 2000 y el 2014 muestran que mayoritariamente en España se encuentran residiendo en la actualidad personas extranjeras de nacionalidad rumana (casi el $20 \%$ de los extranjeros), seguido de marroquíes (entorno al $15 \%$ ), y británicos, italianos y ecuatorianos, entorno al 5 $\%$, cada uno de ellos. Si atendemos a la evolución de las mismas, en lo últimos 15 años destacarían varias cuestiones: marroquíes y británicas son las poblaciones extranjeras más estables en número a lo largo del periodo considerado (entre 15 y $20 \%$ los primeros y entre 5 y $8 \%$ los segundos), dado que siempre aparecen entre las cinco nacionalidades más numerosas; la población rumana se hace muy presente a partir de su entrada en la Unión Europea en 2007, ello se explicaría en gran medida porque el estatus de sus ciudadanos cambia de no comunitarios a comunitarios (exención de visado); a principios 
de siglo las nacionalidades mayoritarias en España eran europeas, a excepción de la presencia histórica de marroquíes en España; y con respecto a las nacionalidades de algún país de Centro y Sur América, sin duda, la ecuatoriana es la más visible en todo el periodo, seguida de la colombiana. Pero hay algo que no se percibe atendiendo solo a las cinco nacionalidades más numerosas en todo el periodo, y es que otras nacionalidades de procedencia latinoamericana (peruana y boliviana, principalmente) tienen porcentajes muy parecidos al 5 $\%$, donde en casi todos los años considerados se establece el corte de las cinco nacionalidades más numerosas.

¿Qué sucede cuando observamos el fenómeno de la inmigración y la extranjería en la escuela? Desde hace tiempo venimos observando desde nuestro grupo de investigación esta realidad (pueden consultarse nuestros trabajos más recientes en García et al.2013, 2015), y ello nos ha permitido constatar ciertas particularidades, debidas sin duda a la franja de edad que observamos cuando hablamos de inmigración, extranjería y escuela. Ahora bien, en los últimos 15 años se constata que el alumnado de nacionalidad extranjera ha aumentado desde un $1,5 \%$ del total de los escolares hasta casi un $10 \%$ hace cinco años, pero que en la actualidad la población escolar extranjera ha dejado de crecer. Véase la Tabla 3:

Tabla 3. Evolución población extranjera matriculada en enseñanzas generales

\begin{tabular}{|c|c|c|}
\hline Curso escolar & Número de escolares & $\%$ \\
\hline $1999-2000$ & 103.717 & 1,5 \\
\hline $2004-2005$ & 449.936 & 6,5 \\
\hline $2009-2010$ & 734.008 & 9,6 \\
\hline $2014-2015$ & 668.186 & 8,3 \\
\hline
\end{tabular}

Fuente: elaboración propia a partir de las Estadísticas de Educación (Ministerio de Educación, Cultura y Deporte).

Con respecto a las nacionalidades más numerosas en la escuela es preciso hacer notar que son distintas con respecto a la población 
en general. Así, según datos del Ministerio de Educación, Cultura y Deporte para el periodo 2000-2014, marroquí, rumana y algunas de las nacionalidades de países latinoamericanos (ecuatoriana, colombiana, etc.) presentan porcentajes parecidos -sobre el $20 \%$-, pero la población británica, muy presente en los datos generales, desaparece de las estadísticas educativas donde solo son visibles de manera importante $(6,04 \%$ ) en el curso 1999/2000 (siendo su presencia para el resto de los años menor al $5 \%$ ). Ello responde a las características de esta población en España, que -en muy alto grado- son personas jubiladas y no familias jóvenes con hijos en edad escolar. Es interesante también ver cómo emergen otras nacionalidades de países latinoamericanos, que no estaban presentes en las estadísticas a nivel general, como pueden ser la argentina, la boliviana y la dominicana. La población extranjera con nacionalidad de algún país de Sur y Centro América es, como decíamos al inicio de este texto, el segundo grupo por orden de importancia si atendemos a la procedencia continental (por detrás de quienes ostentan nacionalidad de algún país africano). Sin embargo, mientras que esta última son en su gran mayoría marroquíes, la población identificada como "latinoamericana" son de muy diversas procedencias.

Exponemos a continuación las representaciones e imaginarios sociales que existen alrededor de estos grupos y colectivos, y cómo las mismas están contribuyendo a la generación de lógicas de construcción de la diferencia en la escuela.

\section{Alteridad y representaciones sociales sobre el alumnado "latinoamericano"}

Podemos encontrar que las representaciones sociales sobre el alumnado "latinoamericano" en la escuela española responden, por un lado, a la imagen y estereotipos que existen sobre la inmigración en su conjunto en el país, lo cual coincide con los imaginarios y lógicas de construcción de la diferencia hacia los inmigrantes que funcionan en casi 
todos los países pertenecientes a la Unión Europea ${ }^{5}$ y por otro, a la idea que existe en el país sobre "Latinoamérica" en general (y aquí es donde encontramos vestigios de la época colonial).

\section{Construcciones socioculturales, inmigración y escuela ${ }^{6}$}

Apuntábamos al inicio de este texto cómo los movimientos migratorios actuales llevan tiempo siendo definidos por medios de comunicación, discursos políticos y sociedad en su conjunto como una problemática social de primer orden. Ello implica que, en las sociedades globalizadas, se ha construido una imagen del inmigrante extranjero como el "otro por excelencia", como el "summun de la otredad" (Olmos-Alcaraz, 2012). ¿Pero qué dimensiones son las que integran tal representación del inmigrante extranjero? En primer lugar el inmigrante extranjero es visto como "extraño", "lejano" y "distante" (Simmel, 2002; Bauman, 2005) pero que habita con el nosotros; y es definido también como inferior: persona sin recursos, pobre, distante culturalmente y delincuente (Santamaría, 2002; Rea, 2006; Van Dijk, 2009).

Esta imagen se reproduce a pequeña escala -aunque con algunos matices- si observamos las representaciones que se hacen del alumnado extranjero en tanto inmigrante:

Existe una imagen del/la alumno/a inmigrante extranjero/a como un sujeto portador y generador de dificultades y contrariedades, hasta el punto de servir para explicar la existencia de problemas que ya estaban presentes en la escuela antes de su incorporación,

5 Dicha coincidencia es debida en parte a que es la propia Unión Europea quien, a través de su política migratoria, ha contribuido y contribuye a la creación de una imagen de la inmigración extra-comunitaria como problema (Olmos-Alcaraz, 2009; Sebastiani, 2015).

6 Mantendremos el anonimato de las personas que han sido entrevistadas en nuestras investigaciones, omitiendo sus nombres e identificando los discursos que citamos con el cargo que ocupa la persona y la etapa educativa en la que desempeña sus labores. 
pero que ahora son reinterpretados a la luz de su presencia. (...) El alumnado inmigrante extranjero es percibido y representado principalmente como un alumno que carece de recursos económicos, que tiene dificultades de aprendizaje y que tiene una cultura radicalmente diferente a la "nuestra", con todo lo que ello conlleva (Olmos-Alcaraz, 2012, pp. 76).

De todas las características destacadas para construir a este alumnado como "otro" enfatizamos la importancia otorgada a las "diferencias culturales", porque ello se usa para justificar y explicar todas las desigualdades que les afectan y que no pueden ser explicadas por la clase social o cuestiones individuales (Olmos-Alcaraz, 2012). Ello, en sí mismo, supone una lógica de funcionamiento del racismo muy particular, identificable como "racismo cultural" (Wieviorka, 2006) y específica de las sociedades occidentales actuales globalizadas.

Pero ¿qué acomodo tienen dentro de estas representaciones genéricas el alumnado inmigrante identificado como "latino" o "latinoamericano"? Para ello ofrecemos a continuación un análisis de los imaginarios sociales que -entendemos- están en la base de dichas construcciones socioculturales.

\section{Imaginarios coloniales en contextos escolares: "Latinoamérica" vista desde España}

El nacimiento de la nación española se asienta sobre dos mitos fundacionales: el de la "Reconquista" y el del "Descubrimiento"

"Reconquista" y "Descubrimiento" son los dos mitos de origen de la "nación española". Para la historiografía oficial, que responde

7 Mitos que además podemos calificar de eufemismos: la "Reconquista" fue más bien una conquista y el Descubrimiento, colonización. 
al discurso del nacionalismo de estado españolista, ambos "hechos" -en realidad, ambos supuestos hechos- son los generadores de España como "Estado-Nación” (...) para el discurso oficial (...) son hechos históricos evidentes en sí mismos, incuestionables, que no necesitan argumentarse ni admiten discusión alguna. (Moreno, 1992, pp. 56-57).

Es a partir de los mismos que se formaron muchas de las representaciones sociales sobre el "otro" cultural, exterior, no nacional, extranjero, en España. Con respecto al primero, la "Reconquista", la diferenciación se estableció especialmente por motivos étnico-religiosos generando una idea sobre los árabes-musulmanes como enemigos, como <<salvajes>>, fanáticos y crueles, deseosos de venganza tras ser expulsados de la Península Ibérica (ver Martin, 2004). En el caso del "Descubrimiento" el proceso de alterización acontecido fue distinto, y referido a la población del "Nuevo Mundo" a partir de marcadores étnicos exclusivamente, dado que se consigue la evangelización de buena parte de la misma. El resultado fue una imagen del "otro", en este caso, como el $<<$ buen salvaje $>$, en tanto que alguien que "vive en estado de naturaleza, casi como un niño, a la espera de ser civilizado" (Olmos-Alcaraz, 2016b). Un "otro" visto y construido desde un enfoque paternalista como infantil y atrasado (Rubio-Gómez, 2013):

Con perfecto derecho los españoles imperan sobre estos bárbaros del Nuevo Mundo, los cuales en prudencia, ingenio, virtud y humanidad, son tan inferiores a los españoles como los niños a los adultos y las mujeres a los hombres. (Ginés de Sepúlveda, citado en Moreno, 1992, p. 59).

Este mito fundacional de la nación española sigue estando incrustado en los imaginarios que a día de hoy aún funcionan en el país y que tienen que ver con la época colonial. Para avanzar un poco en las características de dichas representaciones sociales es preciso reparar en cómo es construida la idea de América Latina desde este 
contexto $^{8}$, que hace tabula rasa de todo lo existente previo a la colonización; es decir, es preciso pensar en cómo "Latinoamérica" es vista desde España.

En este sentido, lo que primero salta a la vista es quizá la propia denominación de los contextos sobre los que estamos pensando. Al hablar de América Latina o "Latinoamérica" se olvida que esta terminología alude a una adscripción de tintes coloniales creada por las élites criollo-mestizas y que refleja justamente su visión geopolítica del mundo (Mignolo, 2007). Esta categorización excluye de raíz a cualquiera de los pueblos y etnias originarias de las Américas, que no tienen mucho de "latinos" a excepción de la lengua y la religión que se les impuso, y que fueron -cómo no- re-interpretadas a lo largo del proceso de aculturación forzosa al que fueron sometidos. En este sentido, en primer lugar, destacamos un proceso de homogeneización y reducción de las diversidades en cómo "Latinoamérica" es vista desde España. Hablamos, claro está, desde nuestros espacios de trabajo y de producción de conocimiento: los espacios educativos formales. Pero sospechamos que existe un gran paralelismo entre lo producido en la escuela y lo existente en la sociedad en general. Dicha homogeneización se articula, por un lado, con respecto a cuestiones idiomáticas, entendiéndose que todos aquellos que habitan/proceden en/de este vasto territorio cuentan con "una lengua materna derivada del latín, lo que supone ignorar a más de 25 millones de personas cuyas lenguas se identifican como indígenas” (Rubio-Gómez, 2013, pp. 249).

Por otro lado se atribuye una supuesta identidad que gira entorno a la idea de "latinidad" como etnónimo, que de nuevo homogeneiza y asimila a poblaciones indígenas y no indígenas, a poblaciones pertenecientes a distintos grupos étnicos y que incluso desconoce o se sorprende ante la existencia de poblaciones descendientes de esclavos negros procedentes de África, en tanto que una parte más de la diversidad que alberga dicho territorio:

“(...) El profesorado todavía no tiene muy claro qué diferencias hay entre un alumno inmigrante y otro que no..., de hecho te puedo

8 Para un mayor desarrollo de este punto ver Rubio-Gómez (2013). 
comntar un caso muy curioso: he ido a un colegio el otro día, he preguntado si había alumnos inmigrantes porque era de mi zona, de aquí, de Cádiz; y me dijeron que no había ninguno, que no había nadie. Y cuando salí, un niño negro, negro como el..., además que tenía la pinta a primera impresión de que era cubano. Me vuelvo y le digo: - “'Tenéis algún niño cubano o algo así??”- - “Sí, es verdad, tenemos dos cubanos”.- -Pero eso son inmigrantes ¿no?-“. (Técnico de la Administración Educativa).

Y que se construye en oposición a la América Sajona o de habla inglesa (Ardao, 1993).

Esta idea de "latinidad", de "Latinoamérica", reflexionada a la luz de los fenómenos migratorios actuales en contextos educativos formales se materializa en la construcción de un figura de alteridad que alberga las características del mito del <<buen salvaje>>, en este caso, con respeto al alumnado. Son tres los elementos definitorios que destacamos en ello: atraso, bondad y similitud cultural. En la Tabla 4 ofrecemos un desarrollo más amplio de estos tres elementos, que resumen cuáles son las representaciones y los discursos existentes sobre el alumnado denominado "latinoamericano":

Tabla 4. Representaciones y discursos sobre el alumnado denominado "latinoamericano" en la escuela española

\begin{tabular}{|c|c|c|}
\hline $\begin{array}{c}\text { “Atraso" social, económico } \\
\text { y cognitivo }\end{array}$ & Bondad innata & Similitud cultural \\
\hline $\begin{array}{c}\text { Escolarización previa en sistemas } \\
\text { educativos de bajo nivel }\end{array}$ & $\begin{array}{c}\text { Buenas } \\
\text { personas }\end{array}$ & Lengua \\
\hline Desinterés personal y familiar & Alegres & Religión \\
\hline \begin{tabular}{rl|l} 
Preferencia por el trabajo y no por \\
el estudio
\end{tabular} & Sociables & \\
\hline Absentismo escolar y abandono & Dóciles & \multirow{2}{*}{ Otros rasgos } \\
\hline Perezosos & Obedientes & \\
\hline Coeficiente intelectual bajo & Respetuosos & \\
\hline
\end{tabular}

Fuente: elaboración propia a partir de Rubio-Gómez (2013) y análisis de datos de campo. 
Sería necesario analizar cada uno de los tres elementos señalados, si bien no tenemos espacio para desarrollar en detalle todos los matices y particularidades encontradas en los discursos y prácticas indicados. Remitimos para ello a Rubio-Gómez (2013).

Con respecto a esa percepción de "atraso" social, económico y cognitivo, a lo largo de nuestros trabajos de campo, encontramos que tanto en los niveles de educación primaria como secundaria, el profesorado entendía que el alumnado "latinoamericano" procedía de contextos que determinaban de alguna manera la existencia de un menor interés hacia la educación escolar:

"Yo creo en sus países la enseñanza no es algo común... no sé, vienen de zonas en las que a lo mejor es difícil acceder a la enseñanza, y a lo mejor por eso ellos no están motivados por el aprendizaje, sobre todo los que vienen aquí". (Profesor de ATAL, Centro de Educación Secundaria).

Y eso era, según ellos, lo que explicaba que tuviesen itinerarios escolares entendidos como deficitarios. Aunque también encontramos que una idea de los mismos como carentes de recursos económicos, sobre todo en sus países de origen, era esbozada para justificar que en la escuela "perdiesen el interés", dado que a su llegada a España "lo tenían todo" y eso les abrumaba. Así lo explicaba una profesora de apoyo:

\footnotetext{
"Yo desde mi experiencia [hablando de alumnado ecuatoriano] y es lo que veo es que estos niños vienen de países muy necesitados, donde hay muchas carencias y claro, vienen aquí y 'angélicos', pues pasan de tener nada a tenerlo todo... y se desbordan" (Profesora de Apoyo, Centro de Educación Secundaria).
}

Encontramos además como a veces algunas nacionalidades "quedaban exentas" de estas clasificaciones, el caso del alumnado argentino, que -como podemos ver en la cita que sigue- es percibido con una mayor cercanía al "nosotros". Véase también como la profesora entrevistada habla de ecuatorianos, bolivianos y colombianos como alumnos que 
"no son trabajadores" y a los que "les cuesta mucho aprender", a pesar de conocer el idioma:
“(...) No hacen nada ni en clase, son niños que les cuesta mucho incluso a pesar de conocer el idioma, el nivel que traen es que les cuesta, les cuesta mucho, le tienes que repetir, repetir y repetir para alcanzar unos niveles mínimos, hay algunos que han sido educa- dos con el azote y otros bueno, tendrán que repetir este año y a ver el curso que viene. Si te fijas no encontrarás ningún latinoa- mericano en bachillerato, bueno argentinos sí, pero esos son más como nosotros, me refiero a ecuatorianos, bolivianos, colombia- nos, etc.”. (Profesora de Lengua Castellana y Literatura, Centro de Educación Secundaria).

Son representados como niños que son "lentos" y que "no dan más de sí”. Incluso se apunta a que no es una cuestión individual, sino que tiene que ver con los países de origen, cuando -como puede apreciarse la cita que sigue- el profesor dice "no sé cómo será la educación en su países, pero vienen con mucho retraso".
“(...) Son casi todos latinoamericanos, y estos niños son muy len- tos, muy muy lentos. No sé cómo será la educación en sus países, pero vienen con mucho retraso y es como que su cerebro va más lento que el nuestro. Parece que sus esquemas cerebrales no dan más de sí. Les tengo que explicar muy despacio, y luego otra vez, y otra vez, y luego les hago el control y no lo han aprendido. Y después del verano no se acuerdan de nada". (Maestro, Centro de Educación Primaria).

La idea de retraso, precisamente, está en la base del imaginario sobre "lo latinoamericano", y sustenta las representaciones del "otro" como sujeto infantil, puro y en estado de naturaleza.

En lo relativo a la construcción del alumnado latinoamericano como "bueno por naturaleza" queremos señalar especialmente la percepción de "docilidad”. Foucault afirmó que "es dócil un cuerpo que puede ser sometido, que puede ser utilizado, que puede ser transformado 
y perfeccionado" (2002, pp. 108). De la siguiente manera hablaba una profesora de Educación Física sobre los alumnos "latinoamericanos", alabando que son alumnos de buen comportamiento:

"Para mí todos son iguales pero quizá los que menos problemas tienen aquí son los latinoamericanos porque realmente se comportan muy bien, son muy nobles y serviciales... otra cosa es a nivel académico... pero integrarse, se integran estupendamente" (profesora de Educación Física, Centro de Educación Secundaria).

$\mathrm{Al}$ igual que este Director de un Centro de Educación con niveles de Primera y Secundaria, que de nuevo ofrece una imagen -que para él es- positiva de este alumnado, destacando sus formas de hablar y relacionarse con el profesorado, en tanto que muy respetuosas:

"A mí me sorprende y me gusta como hablan, todo lo piden "por favor., siempre de usted... yo sí que diría que los latinoamericanos están casi mejor educados que los nuestros, los españoles. Porque aquí el profesorado de lo que se queja es de la mala educación de los niños, de la falta de respecto, y en eso, por lo menos aquí, en el centro son peores los nacionales, los españoles, que los extranjeros, o sea que los problemas del día a día de la disciplina y de eso los tenemos aquí más con los españoles que con los extranjeros" (Director, Centro de Educación Primaria y Secundaria).

En comparación con lo que él define "los nuestros, los españoles".

Y el aspecto más controvertido que hemos encontrado en nuestras investigaciones es quizá el que tiene que ver con el imaginario social de alumnado "latinoamericano" como muy similar al "nosotros". Decimos controvertido porque opera discrecionalmente: emerge cuando refuerza aspectos positivos del "nosotros" y se diluye cuando puede ser relacionado con cuestiones negativas del endo-grupo. Veámoslo con dos ejemplos: la similitud/diferencia idiomática y la similitud/diferencia cultural (entendida ésta en términos de idiosincrasia, valores $\mathrm{y}$ formas de ser). 
El director de un Centro de Educación Secundaria, al ser entrevistado sobre el tipo de alumnado inmigrante extranjero que había en su centro, nos describía al alumnado "latinoamericano" como más integrable que otro alumnado de otras nacionalidades o procedencias; pero además también "más aceptado", destacando en ello precisamente que hablan español:

"Es que son muy alegres, hablan con todo el mundo y eso hace que se integren mejor que otros alumnos que no hablan español. Yo diría que por regla general los alumnos aceptan bien a los latinoamericanos, ya están acostumbrados... en realidad yo creo que ya el alumnado está acostumbrado a que va llegando alumnado de todo tipo, no solamente inmigrantes, y que si vienen estos y hablan español pues siempre es mucho más fácil que se integren" (Director, Centro de Educación Secundaria).

Vemos aquí el proceso de homogeneización etnocéntrico, mencionado más arriba, y que obvia la gran cantidad de formas dialectales derivadas del castellano usadas en América Sur y Centro (Rubio-Gómez, 2013).

Por otro lado, esta misma característica es vista por otros como algo que nos diferencia, a españoles y "latinoamericanos". Así lo manifestaba un profesor de matemáticas:

"Mucho mejor sería que primero aprendieran español, ojo, que aunque sean hispanohablantes muchos... mira el chaval este ecuatoriano, yo no sé si es que allí hablan de otra manera concretamente diferente, pero ese se entera de poco y tienen algunos giros... claro que sí, yo no los entiendo a ellos lógicamente tampoco me van a entender a mí, se aburren, esta gente se aburren y dejan de estudiar. Cumplen los 16 años y adiós" (profesor de Matemáticas, Centro de Educación Secundaria).

Aludiendo a que "la lengua no es la misma", argumentando que, de hecho, es tremendamente diferente a la "nuestra" cuando no se puede justificar de otro modo que nuestro sistema educativo no logra atender la diversidad que alberga, cuando no logra construir itinerarios de éxito 
para este alumnado. En este caso, el problema es que su lengua no es "realmente la nuestra", si lo fuera -se entiende-, no tendrían problemas. Véase el etnocentrismo que opera en esta lógica de razonamiento.

Y sucede algo muy parecido cuando las representaciones sociales están relacionadas con lo que se esboza como "similitud cultural" entre "ellos" y "nosotros", pero que a veces parece que era más patente en un pasado "indeterminado". En este caso citamos un fragmento de entrevista de un profesor de Lengua Castellana y Literatura que, refiriéndose a este alumnado como "hispanoamericanos" destaca que vienen con "valores tradicionales" que les hacen ser respetuosos con las familias, valores que pierden al relacionarse con los niños españoles (idea del "buen salvaje", en estado de naturaleza que se pervierte al entrar en contacto con los aspectos menos positivos del "progreso"):
“(...) Los hispanoamericanos lo que sí encuentras sobre todo cuan- do hablas con los padres de estos niños es que los valores fami- liares y valores tradicionales que ellos traen aquí desaparecen y los problemas que están teniendo los padres con sus hijos son que pierden el respeto familiar (...) ese es el gran choque que están te- niendo y se quejan, se quejan de eso de que vienen con unos va- lores que aquí, con el contacto de sus hijos con otros niños de su misma edad hacen que los pierdan y se sientan muy incómodos" (profesor de Lengua Castellana y Literatura, Centro de Educación Secundaria).

Este discurso representa un imaginario que destaca que el alumnado "latinoamericano" tiene cosas buenas, civilidad, valores tradicionales y familiares, que "nosotros" les dejamos y ahora nosotros hemos perdido por el tipo de vida "moderna" que vivimos. Se trata de valores que -se entiende- pertenecen a nuestro pasado, y que perdimos con el avance de la sociedad y que ahora regresan de la mano de poblaciones inmigrantes entendidas como cercanas, próximas, familiares... al tiempo que construidas como desfasadas y anticuadas (Rubio-Gómez, 2103; García y Rubio-Gómez, 2013).

Pero, como decíamos, no hay un acuerdo en este sentido. Las representaciones sobre las características culturales de estas poblaciones 
escolares son ambivalentes, contradictorias si se quiere, porque también encontramos que se destaca la "diferencia cultural" entre un "ellos" y un "nosotros". Una profesora de apoyo nos contaba que, según ella, las familias "latinoamericanas" son familias desestructuradas (en vez de decir que las familias en la sociedad actual son de tal manera, y como si las españolas no lo fuesen), y destacaba este rasgo para poner de manifiesto que los niños estaban de algún modo "abandonados":
“(...) Todas o casi todas las familias latinoamericanas son familias desestructuradas, y más que desestructuradas es no organizadas, no organizadas, entonces es que el padre trabaja, la madre trabaja, llegan cansados, tienen que hacer la comida, tiene que hacer esto, lo otro, pero los niños están ahí y nadie les hace caso" (profesora de apoyo, Centro de Educación Secundaria).

Un orientador de un Centro de Educación Secundaria lo manifestaba aún más claro, al aludir que es por su "cultura" que este alumnado no estudia, abandonan sus itinerarios de formación en las etapas no obligatorias (de nuevo, como si los españoles no operaran de igual manera):

"Ellos tienen su cultura y los padres lo que quieren, en el caso de
los ecuatorianos, es que cuando el niño cumpla 16 años se pongan
a trabajar porque es lo que le toca. A partir de los dieciséis años
como ya no es obligatoria la asistencia están deseando cumplirlos
para dejar de venir. Pero eso es así, están deseando cumplir los 16
años para ponerse a trabajar porque culturalmente no le ven sali-
da a los estudios" (Orientador, Centro de Educación Secundaria).

Por último, decir que es el profesorado que trabaja directamente con el alumnado y no la dirección de los centros o la inspección educativa, orientadores y otras figuras que trabajan en los centros quienes ven más diferencias y menos cercanía entre el alumnado latinoamericano y el alumnado español, y los imaginarios sociales sobre la cercanía cultural son más frecuentes entre quienes no tienen un trato tan cercano y directo (Rubio-Gómez, 2013). 


\section{Reflexiones finales}

Hemos mostrado los discursos más representativos de nuestros trabajos de campo en relación a las representaciones sociales existentes en el momento actual sobre el alumnado inmigrante extranjero, y dentro de este, el alumnado identificado como "latinoamericano". La primera reflexión que nos surge después de realizar esta tarea es que no existe una imagen unitaria, fija u homogénea sobre los mismos. Incluso vemos cómo a veces esos imaginarios entran en contradicción. Si bien la imagen que existe del alumnado inmigrante extranjero en general, en el contexto estudiado, es una imagen que destaca elementos problematizados y negativos sobre otros posibles más neutros o positivos; cuando se particulariza y se emiten discursos sobre lo "latinoamericano" en la escuela los discursos se perfilan y emergen ideas como las señaladas de "bondad innata" o "cercanía cultural" (no sin objeciones) que -entendemos- tienen su origen en la época colonial. Siendo así, el alumnado "latinoamericano", aunque es percibido también de manera problematizada, lo es menos en relación a otros alumnos cuyas procedencias no se conciben tan "cercanas" a la española. En trabajos anteriores hemos mostrado cómo todo ello tiene una traducción dicotómica y ambivalente al mismo tiempo, donde estos alumnos son vistos en la escuela española como "buenos alumnos y malos estudiantes” (Rubio-Gómez y Olmos-Alcaraz, 2013; Olmos-Alcaraz y RubioGómez, 2013): "buenos” en cuanto a comportamiento y acatamiento de las normas escolares no estrictamente curriculares y "malos" con respecto a la consecución de logros académicos esperados.

Una segunda reflexión tiene que ver con el acomodo que están teniendo estas representaciones e imaginarios en la institución escolar española. Mostrábamos cómo es la presencia estadística de las poblaciones migrantes extranjeras en los niveles de educación primaria y secundaria. Los datos nos dicen que los alumnos procedentes de países de Centro y Sur América no son los más numerosos, aunque sí representan un volumen considerable entre los mismos. No obstante la diversidad que añade este alumnado al sistema educativo no supone un objeto de intervención pública prioritario, dado que, en este sentido, las políticas 
educativas de integración son básicamente políticas lingüísticas. El sistema educativo español no se está transformando por la llegada de estos alumnos o -en otras palabras- no está dando respuestas educativas a las diversidades más allá de la lengua. Sin embargo las construcciones de alteridad que hemos mostrado, que existen en las escuelas e institutos estudiados, influyen en los proyectos socio-educativos de estos chicos. Ello está propiciando que se construyan itinerarios de "éxito" e itinerarios de "fracaso" (Rubio-Gómez, 2013), reforzados por los imaginarios de lo que supone ser buen o mal alumno/a, buen o mal estudiante. El gran problema de todo ello es que las explicaciones a estas situaciones -las más de las veces- recaen sobre el propio alumnado y sus familias. Recordemos algunas de las ideas expresadas en las entrevistas citadas más arriba: sus sistemas educativos de origen, su formación previa, sus formas de razonamiento, sus familias desestructuradas... "sus culturas” parecen ser las culpables de todos los males que les afectan. Y es aquí donde detectamos procesos de construcción de la diferencia que se acercan a lógicas de funcionamiento de lo que se conoce como "nuevo racismo" o "racismo cultural" (Wieviorka, 2006), donde una idea de "cultura" esencializada funciona como marcador que genera diferencias irreductibles.

Por último, no podemos sino finalizar recordando la necesidad de seguir implementado programas educativos interculturales porque es a través de los mismos que se podrá trabajar para que las diversidades se pongan en valor y puedan visibilizarse de manera que reviertan en mejoras educativas para todos. Así mismo, incidir en la necesidad de una formación en este sentido para los docentes que se enfrentan a situaciones que les son nuevas y para las que no han sido preparados previamente. Dicha formación habría de ser inicial y continua, pero no centrada exclusivamente en aspectos lingüísticos. Hemos tratado de mostrar en este texto cómo - de este modo- se quedan fuera "muchas diversidades". Se trataría más bien de programas formativos para dotar de herramientas críticas al profesorado, de cara a posibilitar el desarrollo de destrezas para la transformación social (Olmos-Alcaraz, 2016a). En otras palabras, de formar con y en competencias interculturales (Olmos-Alcaraz y Martínez-Chicón, 2014) que propicien la emergencia de procesos de innovación y cambio desde los propios docentes. 


\section{Bibliografía}

Ardao, A. (1993). América Latina y la latinidad. México: UNAM-CC y -DEL:

Bauman, Z. (2005). Modernidad y ambivalencia. Barcelona: Anthropos.

Foucault, M. (2002). Vigilar y castigar. Nacimiento de la prisión. Buenos Aires: S. XXI.

García, F. J.; Fernández, J., Rubio-Gómez, M. y Soto, M. L. (2007). Inmigración extranjera y educación en España: algunas reflexiones sobre el "alumnado de nueva incorporación”. En M.A. Alegre y J. Subirats (Eds.), Educación e inmigración: nuevos retos para España en perspectiva comparada (pp. 309-359). Madrid: Centro de Investigaciones Sociológicas.

García, F. J. y Olmos-Alcaraz, A. (2010). La promoción de la plena integración de los extranjeros por parte de las administraciones públicas: ¿una protección legal para la construcción de una sociedad multicultural? En J. L. Monereo (Dir.), Protección jurídico-social de los trabajadores extranjeros (pp. 111-177). Granada: Comares S.L.

García, F. J. y Pulido, R. (2008). El desarrollo de la Antropología de la Educación en España: razones que explican la casi monográfica mirada a las llamadas minorías étnicas. En M. I. Jociles y A. Franzé (Coords.), ¿Es la escuela el problema?: perspectivas socio-antropológicas de etnografía y educación (pp. 152-180). Madrid: Trotta.

Granados, A. (2013). La inmigración extranjera informada por los media en España. Nuevos apuntes para comprender su representación. En A. Granados (Ed.), La representación de las migraciones en los medios de comunicación (pp. 7-24). Madrid: Trotta.

Jociles, M. I. (2007). Panorámica de la Antropología de la Educación en España: estado de la cuestión y recursos bibliográficos. Revista de Antropología Social, 16, 67-116.

Martín, E. (2004). Maurofobia/islamofobia y maurofilia/islamofilia en la España del siglo XXI. Revista CIDOB d'Afers Insternacionals, 66-67, 39-51.

Mignolo, W. (2007). La idea de América Latina la herida colonial y la opción decolonial. Barcelona: Gedisa.

Ministerio de Educación, Cultura y Deporte (2015). Estadística de enseñanzas no universitarias. Datos avance 2014-15. Madrid: Ministerio de Educación, Cultura y Deporte. 
Moreno, I. (1992). América y el nacionalismo de estado español del IV al V Centenario. Estudios Regionales, 34, 53-78.

Olmos-Alcaraz, A. (2008). Estudiar migraciones desde la Antropología Social: una investigación sobre la construcción social de la alteridad en contextos educativos, Revista Migraciones, 23, 151-171.

Olmos-Alcaraz, A. (2009). La población inmigrante extranjera y la construcción de la diferencia. Discursos de alteridad en el sistema educativo andaluz (Tesis Doctoral). Granada: Universidad de Granada.

Olmos-Alcaraz, A. (2010). Construcción discursiva del alumno inmigrante extranjero en Andalucía: el otro en nuestras escuelas. Revista de Educación, $353,469-493$.

Olmos-Alcaraz, A. (2012). Cuando migrar se convierte en estigma: un estudio sobre construcción de alteridad hacia la población inmigrante extranjera en la escuela. Imagonautas. Revista Interdisciplinaria Sobre Imaginarios Sociales, 1(2), 62-85.

Olmos-Alcaraz, A. (2016a). Racismo, racialización e inmigración: aportaciones desde el enfoque descolonial para el análisis del caso español. (En prensa).

Olmos-Alcaraz, A. (2016b). Diversidad lingüístico-cultural e interculturalismo en la escuela andaluza: un análisis de políticas educativas. RELIEVE, Revista Electrónica de Investigación y Evaluación Educativa, 22 (2), 1-19.

Olmos-Alcaraz, A. y Martínez-Chicón, R. (2014). Plataformas virtuales e innovación docente universitaria: Affordance de una Guía de Trabajo Autónomo en Antropología para trabajar competencias de intervención en contextos de diversidad. Revista de Educación a Distancia, 42, 1-15.

Olmos-Alcaraz, A. y Rubio-Gómez, M. (2013). Corporalidad del «buen estudiante»: representaciones de género, «raza», etnia y clase social en la escuela española. Revista Iberoamericana de Educación, 62, 163-179.

Rea, A. (2006). La europeización de la política migratoria y la transformación de la otredad. Revista Española de Investigaciones Sociológicas, 116, 157-184.

Rubio-Gómez, M. (2013). Construyendo diferencias desde retóricas de la igualdad. El caso del alumnado denominado latinoamericano en la etapa de educación secundaria obligatoria (Tesis doctoral). Universidad de Granada: Granada.

Rubio-Gómez, M. y Olmos-Alcaraz, A. (2013). Construcción social del “mal estudiante" en la escuela pública española: intersecciones de género, 
"raza”/etnia y clase social. Diálogos sobre Educación. Temas actuales en investigación educativa, 7, 1-19.

Santamaría, E. (2002). La incógnita del extraño. Una aproximación a la significación sociológica de la «inmigración no comunitaria». Barcelona: Anthropos.

Sebastiani, L. (2015). La colonialidad del poder y del saber en las políticas públicas de la Unión Europea: reflexiones a partir de la una investigación sobre "inmigración" e "integración”. Revista de Antropología Experimental, 15, 535-552.

Serra, C. (2004). Etnografía escolar, etnografía de la educación. Revista de Educación, 334, 165-176.

Simmel, G. (2002 [1908]). El extranjero como forma sociológica. En E. Terrén (Ed.), Razas en conflicto. Perspectivas sociológicas (pp. 59-65). Barcelona: Anthropos.

Van Dijk, T.A. (2009). Discurso y poder. Barcelona: Gedisa.

Wieviorka, M. (2006). La mutación del racismo. Migraciones, 19, 151-163.

Zapata-Barrero, R. (2001). Fundamentalismo estatal de la UE en torno a la inmigración. Revista CIDOB d'afers internacionals, 53, 149-176. Recuperado de: http://www.cidob.org/content/download/58485/1519090/.../1/.../ 53zapata.pdf 